\title{
TORSION AND PROTORSION MODULES OVER FREE IDEAL RINGS
}

\author{
P. M. COHN
}

(Received 16 April 1969)

\author{
For Bernhard Hermann Neumann on his 60th birthday \\ Communicated by B. Mond
}

\section{Introduction}

Free ideal rings (or firs, cf. $[2,3]$ and $\S 2$ below) form a noncommutative analogue of principal ideal domains, to which they reduce in the commutative case, and in [3] a category $T_{R}$ of right $R$-modules was defined, over any fir $R$, which forms an analogue of finitely generated torsion modules. The category $T_{R}$ was shown to be abelian, and all its objects have finite composition length; moreover, the corresponding category ${ }_{R} T$ of left $R$-modules is dual to $T_{R}$.

A natural problem at this point is to find an analogue of torsion modules that are not necessarily finitely generated. There are a number of ways of forming the completion of an abelian category (cf. e.g. Gabriel [5]); in our case the problem is simplified by the fact that $\boldsymbol{T}_{R}$ is a full subcategory of $\boldsymbol{M}_{R}$, the category of all right $R$-modules. On the other hand, we shall want an explicit description of the torsion modules and this is not provided by the general theory. We shall therefore proceed somewhat differently: We begin by defining general torsion modules over a fir $(\S 2)$, and show that these modules form the objects of a full subcategory $\boldsymbol{T}_{R}^{\dagger}$ of $\boldsymbol{M}_{R}$ which is abelian and has exact direct limits and a generator, i.e. it is a Grothendieck category. It is obtained from $\boldsymbol{T}_{R}$ by forming direct limits in $\boldsymbol{M}_{R}$.

A dual procedure leads to the notion of a protorsion module, and in this way we obtain a category $\boldsymbol{T}_{R}^{\downarrow}$ with exact inverse limits and a cogenerator. Further, when the categories of left $R$-modules ${ }_{R} T^{\uparrow},{ }_{R} T^{\downarrow}$ are defined correspondingly, we find that there is a duality between $T_{R}^{\uparrow}$ and ${ }_{R} T^{\downarrow}$. This duality allows us to dispense with the verification that $T_{R}^{\downarrow}$ is again an abelian category. Using a result of Gabriel [5], we can interpret $T_{R}^{\downarrow}$ as the category of all pseudo-compact modules over a certain pseudo-compact ring $E$. In the special case of a principal ideal domain, $E$ can be constructed as a completion of $R$ (e.g. when $R=\boldsymbol{Z}, E=\hat{\boldsymbol{Z}}$ ), but this construction does not extend to general firs.

\section{The category of general torsion modules over a fir}

All rings are understood to have a unit-element 1 , modules are unital and homomorphisms preserve 1. By a free right ideal ring, or right fir for short, one un- 
derstands a ring in which all right ideals are free, of unique rank; left firs are defined correspondingly. Throughout this note, we shall only be dealing with rings that are left and right firs.

Given a fir $R$, and an $R$-module $M$ with a finite presentation

$$
0 \rightarrow R^{m} \rightarrow R^{n} \rightarrow M \rightarrow 0,
$$

the characteristic of $M$ is defined by

$$
\chi(M)=n-m .
$$

If $M$ is finitely generated but not finitely related, we put $\chi(M)=-\infty$, and if $M$ is not finitely generated, $\chi(M)=\infty$. This defines $\chi(M)$ for every $R$-module, and it is clear that the definition is independent of the presentation chosen for $M$. In [3] it was shown that for any short exact sequence

$$
0 \rightarrow M^{\prime} \rightarrow M \rightarrow M^{\prime \prime} \rightarrow 0
$$

of modules over a fir, we have

$$
\chi(M)=\chi\left(M^{\prime}\right)+\chi\left(M^{\prime \prime}\right),
$$

with the usual conventions about $\infty$ and the rules

$(\alpha)$ if $\chi\left(M^{\prime \prime}\right)=\infty$, then $\chi(M)=\infty$,

$(\beta)$ if $\chi\left(M^{\prime \prime}\right)=-\infty$, and $\chi\left(M^{\prime}\right)=\infty$, no conclusion can be drawn.

In particular, (3) is meaningful whenever $M^{\prime}$ and $M^{\prime \prime}$ are finitely generated.

A torsion module over $R$ was defined in [3] as an $R$-module $M$ such that

(i) $\chi(M)=0$

and either of the following conditions (equivalent when (i) holds):

(ii) for every submodule $M^{\prime}$ of $M, \chi\left(M^{\prime}\right) \geqq 0$,

(iii) for every quotient module $M^{\prime \prime}$ of $M, \chi\left(M^{\prime \prime}\right) \leqq 0$.

Any torsion module in the sense of this definition is necessarily finitely generated; we shall generalize this notion by making the following

Definition. A right $R$-module $M$ is said to be a torsion module if every finite subset of $M$ is contained in a finitely generated torsion module.

We denote the full subcategory of $\boldsymbol{M}_{\boldsymbol{R}}$ whose objects are the torsion modules by $T_{R}^{\dagger}$ and as in [3] denote by $T_{R}$ the subcategory of finitely generated torsion modules. We remark that general torsion modules can be defined without referring back to the finitely generated case by the conditions

(i') every finite subset is contained in a submodule $T$ such that $\chi(T)=0$, and

(ii') for every finitely generated submodule $M^{\prime}, \chi\left(M^{\prime}\right) \geqq 0$.

Clearly (ii') actually holds for all submodules, finitely generated or not. 
Conditions (i') and (ii') again imply a condition on the finitely generated quotient modules, but it is not clear whether this can actually replace (ii'). Before proving it we note a general property of finitely related modules which is used repeatedly in what follows.

LEMMA 2.1. Let $R$ be any ring and $M$ an $R$-module with a presentation

$$
0 \rightarrow G \rightarrow F \rightarrow M \rightarrow 0
$$

where $F$ is free and $G$ is finitely generated. Then $M$ is the direct sum of a finitely presented module and a free module.

This result follows by taking a finite generating set of $G$, expressing it in terms of a basis of $F$ and noting that only finitely many elements of the basis of $F$ are involved.

PROPOSITION 2.2. Let $M$ be any torsion module over a fir. Then

(iii') for every finitely generated or finitely related quotient $M^{\prime \prime}$ of $M, \chi\left(M^{\prime \prime}\right) \leqq 0$.

Proof. When $M$ is finitely generated, $\chi(M)=0$ and the result is clear by (3). Now let $M$ be a general torsion module and $M^{\prime \prime}=M / M^{\prime}$ a finitely generated quotient. Then $M$ contains a finitely generated submodule $N$ such that $N+M^{\prime}=M$. Enlarging $N$ if necessary we may assume that $N$ is a finitely generated torsion module and hence satisfies (iii'). Now $M^{\prime \prime}=\left(N+M^{\prime}\right) / M^{\prime} \cong N /\left(N \cap M^{\prime}\right)$, and so $\chi\left(M^{\prime \prime}\right) \leqq 0$. Thus every finitely generated quotient of $M$ has nonpositive characteristic. If $M^{\prime \prime}$ is finitely related but not finitely generated, then by the lemma, $M^{\prime \prime}=M_{0} \oplus F$, where $F$ is free of infinite rank. Hence $M^{\prime \prime}$ has a finitely generated quotient of positive rank, and so does $M$, but this contradicts what has just been proved.

In order to show that $\boldsymbol{T}_{R}^{\uparrow}$ is an abelian category it is enough to verify

(a) in any short exact sequence (2) of $R$-modules, if any two terms are torsion modules, so is the third, and

(b) for any homomorphism $f$ between torsion modules, both ker $f$ and coker $f$ are torsion modules.

Proof of (a). Let $M^{\prime}, M^{\prime \prime}$ be torsion modules and $X$ a finite subset of $M$. The image of $X$ in $M^{\prime \prime}$ is contained in a finitely generated torsion submodule $T^{\prime \prime}$ of $M^{\prime \prime}$. Pick a finitely generated submodule $L$ of $M$ which contains $X$ and maps onto $T^{\prime \prime}$. Let $L^{\prime}$ be the kernel of the mapping $L \rightarrow T^{\prime \prime}$ (induced by the mapping $M \rightarrow M^{\prime \prime}$ ), then $\chi\left(L^{\prime}\right)=\chi(L)-\chi\left(T^{\prime \prime}\right)=\chi(L)$. The latter is finite, so $L^{\prime}$ is finitely generated, and is therefore contained in a finitely generated torsion submodule $T^{\prime}$ of $M^{\prime}$. Let $T$ be the submodule of $M$ generated by $L$ and the image of $T^{\prime}$ under the mapping $M^{\prime} \rightarrow M$. Then $T$ is an extension of $T^{\prime}$ by $T^{\prime \prime}$, hence a finitely generated torsion module, and it contains $X$.

Next assume that $M$ and $M^{\prime}$ are torsion modules. Given any finite subset 
$X$ of $M^{\prime \prime}$, there exists a finite subset of $M$ mapping onto $X$, and hence a finitely generated torsion module $L$ of $M$ whose image $L^{\prime \prime}$ contains $X$. Let $L^{\prime}=$ $\operatorname{ker}\left(L \rightarrow L^{\prime \prime}\right)$; thus if $M^{\prime}$ is identified with a submodule of $M$, then $L^{\prime}=L \cap M^{\prime}$. Since $\chi(L)=0$ and $L^{\prime \prime}$ is finitely generated, $L^{\prime}$ is finitely related. Suppose first that $L^{\prime}$ is finitely generated and enlarge it to a finitely generated torsion submodule $T^{\prime}$ of $M^{\prime}$. Then $T=T^{\prime}+L$ is finitely generated and hence has non-negative characteristic. But as homomorphic image of the torsion module $T^{\prime} \oplus L$ it has non-positive characteristic, hence $\chi(T)=0$ and $T$ is a torsion module. Now coker $\left(T^{\prime} \rightarrow T\right)$ is a finitely generated torsion submodule of $M^{\prime \prime}$ containing $X$. It remains to consider the case when $L^{\prime}$ is not finitely generated. We know that it is finitely related, so $L^{\prime}=L_{0} \oplus F$, where $L_{0}$ is finitely presented and $F$ is free of infinite rank. Since $M^{\prime} \cap L=L_{0}+F$, we can find a finitely generated torsion submodule $M_{1}$ of $M^{\prime}$ which contains $L_{0}+F_{1}$, where $F_{1}$ is a direct summand of $F$, chosen so that $L_{0}+F_{1}$ has positive characteristic. Clearly $M_{1} \cap L=L_{0}+F_{1}$ and the exact sequence

$$
0 \rightarrow M_{1} \cap L \rightarrow M_{1} \oplus L \rightarrow M_{1}+L \rightarrow 0
$$

shows that $M_{1}+L$ has negative characteristic. But this contradicts the fact that $M$ is a torsion module. So this case cannot occur, and $M^{\prime \prime}$ is therefore a torsion module.

Thirdly, assume $M, M^{\prime \prime}$ to be torsion modules and let $X$ be a inite subset of $M^{\prime}$. Let us take $M^{\prime}$ to be a submodule of $M$, then there exists a finitely generated torsion submodule $T$ of $M$ containing $X$. Put $T^{\prime}=T \cap M^{\prime}$ and $T^{\prime \prime}=$ coker $\left(T^{\prime} \rightarrow T\right)$, then $T^{\prime \prime}$ is finitely generated, a quotient of $T$ and a submodule of $M^{\prime \prime}$, and hence is a torsion module. Therefore $T^{\prime}$ is also a torsion module and it contains $X$. This shows $M^{\prime}$ to be a torsion module, and it completes the proof of (a).

Proof of (b). Let $f: M \rightarrow N$ be a homomorphism of torsion modules, and put $K=\operatorname{ker} f$. Any finite subset $X^{\prime}$ of $M / K$ is the image of a finite subset $X$ of $M$. Let $T$ be a finitely generated torsion submodule of $M$ containing $X$ and consider the isomorphism

$$
(T+K) / K \cong T /(T \cap K) .
$$

The left-hand side contains $X^{\prime}$ and is isomorphic to a submodule of $N$, hence $\chi((T+K) / K) \geqq 0$. But (4) shows that $\chi((T+K) / K) \leqq 0$, hence $(T+K) / K$ is a torsion module containing $X^{\prime}$, and we have shown that $M / K$ is a torsion module. Now part (a), just proved, shows that $\operatorname{ker} f \operatorname{im} f$ and coker $f$ are also torsion modules. Thus $\boldsymbol{T}_{R}^{\dagger}$ is an abelian category and we have established part of

THEOREM 2.3. The category $\boldsymbol{T}_{R}^{\dagger}$ of torsion modules over a fir $R$ is a Grothendieck category, i.e. an abelian category with exact direct limits and a generator.

It is clear that $\boldsymbol{T}_{R}^{\dagger}$ is closed under direct unions, and the AB.5 axiom

$$
\bigcup\left(M_{\alpha} \cap N\right)=\left(\bigcup M_{\alpha}\right) \cap N
$$


follows because we have a subcategory of $M_{R}$. To obtain a generator we simply form the direct sum of one copy each of all the simple finitely generated torsion modules.

\section{Protorsion modules}

Definition. A protorsion module over a fir $R$ is a module of the form

$$
M=\varliminf M_{i},
$$

where $\left(M_{i}\right)$ is an inverse system of finitely generated torsion modules.

Every protorsion module carries a natural topology, obtained by taking finitely generated torsion modules to be discrete and regarding $M$ as a submodule of the topological product $\Pi M_{i}$. We shall write $T_{R}^{\downarrow}$ for the category consisting of all protorsion modules and all continuous homomorphisms between them. By arguments similar to those of $\S 2$ it can be shown that $\boldsymbol{T}_{R}^{\downarrow}$ is an abelian category satisfying the dual of AB.5; this will also follow directly from the next result which establishes a duality between ${ }_{R} \boldsymbol{T}^{\downarrow}$ and $\boldsymbol{T}_{R}^{\dagger}$. Note that this extends the duality between ${ }_{R} \boldsymbol{T}$ and $\boldsymbol{T}_{R}$ described in [3], Th. 5.2. We shall abbreviate $\operatorname{Ext}_{R}^{1}$ by Ext in what follows; this is unambiguous because $R$ is a fixed ring, always a fir, and so $\operatorname{Ext}_{R}^{2}=0$.

THEOREM 3.1. Over any fir $R$, the category $\boldsymbol{T}_{R}^{\dagger}$ of right torsion modules is dual to the category ${ }_{R} T^{\downarrow}$ of left protorsion modules.

Proof. Let $M \in T$, ${ }_{R}^{\dagger}$ say $M=\underline{\lim } M_{i}$, where $M_{i} \in T_{R}$. Then, on writing $D M=\operatorname{Ext}(M, R)$ (tor any $R$-module $M)$, we have

$$
D M=\operatorname{Ext}(M, R)=\operatorname{Ext}\left(\underline{\varliminf} M_{i}, R\right)=\varliminf \operatorname{Ext}\left(M_{i}, R\right)=\underline{\varliminf} D M_{i} .
$$

By Th. 5.2 of [3], $D M_{i} \in{ }_{R} T$, hence $D M \in{ }_{R} T^{\downarrow}$. Thus

$$
D: M \mapsto \operatorname{Ext}(M, R)
$$

is a contravariant functor from $\boldsymbol{T}_{R}^{\uparrow}$ to ${ }_{R} T^{\downarrow}$. We complete the proof by showing that $D$ is a duality. In the first place we observe that $D$ preserves short exact sequences, because $\operatorname{Ext}_{R}^{2}=0$ and $\operatorname{Hom}(M, R)=0$ for any $M \in T_{R}^{\dagger}$. Thus $D$ is exact.

Next we show that every object $P$ of ${ }_{R} T^{\downarrow}$ has the form $D Q$, for some $Q \in T_{R}^{\dagger}$. Let $P=\lim P_{i}$, where $\left(P_{i}\right)$ is an inverse system of modules in ${ }_{R} T$, and write $Q_{i}=$ $D P_{i}$, then $\left(Q_{i}\right)$ is a direct system in $\boldsymbol{T}_{R}$ and hence $Q=\varliminf Q_{i} \in \boldsymbol{T}_{R}^{\dagger}$, so

$$
D Q=D\left(\varliminf_{i}\right)=\varliminf D Q_{i}=\varliminf P_{i}=P .
$$

It only remains to show that for any $M, N \in T_{R}^{\dagger}$, the natural mapping

$$
\operatorname{Hom}(M, N) \rightarrow \operatorname{Hom}(D N, D M)
$$

is a bijection. It is injective, for if $f: M \rightarrow N$ is non-zero, take $M^{\prime} \subseteq M$, finitely 
generated, such that $f M^{\prime} \neq 0$ and choose a finitely generated submodule $N^{\prime}$ of $N$ such that $f M^{\prime} \subseteq N^{\prime}$, then $f$ induces a mapping $f^{\prime}: M^{\prime} \rightarrow N^{\prime}$ which is non-zero; we have the commutative diagram with exact rows

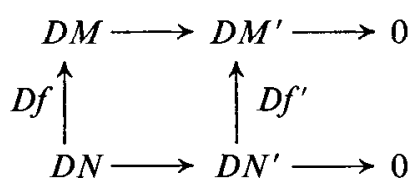

By the duality in the finite case, $D f^{\prime} \neq 0$ and hence $D f \neq 0$.

To show that (2) is surjective, let $g: D N \rightarrow D M$ be given, and assume that $M=\varliminf M_{i}$; then $D M=\varliminf M_{i}$ and by composition we get mappings $g_{i}: D N \rightarrow D M_{i}$. Let $D N=\varliminf_{\alpha} P_{\alpha}$, then by the continuity of $g_{i}$ there exists $\alpha=\alpha(i)$ and a mapping $u_{i}: P_{\alpha} \rightarrow D M_{i}$ such that the triangle

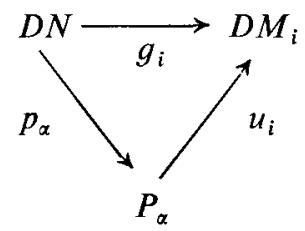

commutes, where $p_{x}$ is the natural projection. Write $Q_{\alpha}=D P_{\alpha}, Q=\lim Q_{\alpha}$, then $D Q=D\left(\lim Q_{\alpha}\right)=\varliminf \lim D Q_{\alpha}=\varliminf P_{\alpha}=D N$, hence $N \cong Q$, by what has been shown. Now $D u_{i}: M_{i} \rightarrow Q_{\alpha}$ combined with the natural injection gives a mapping $u_{i}^{*}: M_{i} \rightarrow Q$. If $\varphi_{j i}: M_{i} \rightarrow M_{j}$ denotes the canonical mapping $(j>i)$, then clearly the $u_{i}^{*}$ satisfy

$$
u_{i}^{*}=u_{j}^{*} \varphi_{j i} \quad \text { for } j>i .
$$

Hence the $u_{i}^{*}$ combine to give a mapping $u^{*}: M \rightarrow Q$ which satisfies $D u^{*}=g$.

It now follows (e.g. by the criterion 1.19 of [1]) that $D$ defines a duality between $\boldsymbol{T}_{R}^{\dagger}$ and ${ }_{R} T^{\downarrow}$, and this completes the proof.

Since the notion of a (two-sided) fir is left-right symmetric, we also have a duality between ${ }_{R} T^{\dagger}$ and $T_{R}^{\downarrow}$.

Let us return to $T_{R}$ and consider $S=\operatorname{Spec} T_{R}^{\uparrow}$, the associated spectral category (cf. $[6,8])$. We recall that the natural functor

$$
P: T_{R}^{\dagger} \rightarrow S=\operatorname{Spec} T_{R}^{\uparrow}
$$

may be described as the solution of the universal problem of making all essential monomorphisms in $T_{R}^{\dagger}$ invertible. Further, $P M \cong P N$ if and only if $M$ and $N$ have isomorphic injective hulls ${ }^{1}$, or equivalently, if there exists $L \in \boldsymbol{T}_{R}^{\dagger}$ with essential monomorphisms $L \rightarrow M, L \rightarrow N$. Consider first the case where $M, N$ are both finitely generated, by $n$ elements say. Writing $T=R_{n}$, the $n \times n$ matrix ring

1 Taken in $T_{R}^{\dagger}$, of course. The injective hull within $M_{R}$ is generally larger. 
over $R$, we may regard $M$ and $N$ as cyclic $T$-modules, say $M=T / c T, N=T / d T$, where $c, d$ are elements of $T$ defining torsion modules. We recall from [3] that an element $c$ defines a torsion module precisely if it has no left or right factor which is a zero-divisor; such an element of $T$ will be called regular. We note that a $T_{R}$-submodule $M^{\prime}$ of $M$ corresponds to a right factor of $c$ : if $c=a b$, then $a T / a b T \cong$ $T / b T=M^{\prime}$ say, $T / a T=M^{\prime \prime}$, where the relation between $M, M^{\prime}, M^{\prime \prime}$ is given by the exact sequence (2) of $\S 1$. In terms of the matrices $a, b, c$ defining $M^{\prime \prime}, M^{\prime}, M$ the monomorphism $M^{\prime} \rightarrow M$ is essential if and only if $b$ is an essential right factor of $c$ in the sense of the following

DEFINITION. Let $c$ be a regular element of a ring $T$. Then a right factor $c^{\prime}$ of $c$ is inessential if $c$ has a non-unit right factor which is right coprime to $c^{\prime}$. Otherwise $c^{\prime}$ is essential.

With this definition two regular $n \times n$ matrices over a fir $R$ correspond to right $R$-modules with isomorphic injective hulls if and only if they have an essential right factor in common.

Since $\boldsymbol{T}_{R}^{\uparrow}$ is locally Noetherian, each injective is a direct sum of indecomposable injectives. In general, these summands are not finitely generated, but any indecomposable injective can be obtained as the injective hull of any one of its non-zero submodules, and we know that there always is a finitely generated submodule.

We recall that an object $P M$ of $S$ is simple if and only if $M$ is coirreducible in $T_{R}$. i.e. if $M$ has a unique minimal non-zero submodule $M^{\prime}$, and clearly $P M \cong$ $P M^{\prime}$. Thus the simple $S$-objects $P$ are just the injective hulls of the $\boldsymbol{T}$-simple torsion modules, so each is determined by an atom in some $R_{n}(n=1,2, \cdots)$. Let $\boldsymbol{P}$ be a system of representatives for the simple $S$-objects, then the mappings $M \rightarrow \operatorname{Hom}_{\boldsymbol{S}}(P, M)(P \in \boldsymbol{P})$ provide an equivalence between $S$ and a product category

$$
\boldsymbol{S} \rightarrow \Pi M_{K_{P}} \text {, where } K_{P}=\operatorname{End}_{\boldsymbol{s}}(P) \text { is a skew field. }
$$

Now in $T_{R}^{\dagger}$ any direct sum of injectives is again injective (cf. e.g. [1], 6.50). Let $U$ be the direct sum of one copy of each indecomposable injective in $T_{R}^{\dagger}$. This module $U$ may also be formed obtained by taking one copy of each $T$-simple module and forming the injective hull. Let $E$ denote the endomorphism ring of $U$ :

$$
E=\operatorname{End}_{T}(U) \text {. }
$$

The ring $E$ may be regarded as a pseudo-compact ring in a natural way ([5], Prop. IV.4.13); by Th. IV.4.4 of [5], ${ }_{R} T^{\dagger}$ is dual to $P C(E)$, the category of pseudocompact right $E$-modules, hence by applying Th. 3.1 above we obtain

THEOREM 3.2. Let PC(E) be the category of pseudo-compact right E-modules, where $E$ is given by (3), then $T_{R}^{\dagger}$ is equivalent to $P C(E)$.

By way of illustration let us briefly consider the case of a principal ideal domain. By [4] the category $\boldsymbol{T}_{R}^{\dagger}$, as subcategory of $\boldsymbol{M}_{R}$, admits submodules if and only 
if $R$ is a principal right ideal domain. In that case the duality of Th. 3.1 reduces to the situation familiar in abelian groups:

$$
\operatorname{Ext}(M, R)=\operatorname{Hom}(M, K),
$$

where $K=I / R, I$ being the injective hull of $R$ as right $R$-module. More particularly, assume that $R$ is a principal ideal domain (not necessarily commutative). Then every simple object in $T_{R}^{\dagger}$ is a cyclic module and hence of the form $R / p R$ ( $p$ an atom of $R$ ). Now $I$ is just the skew field of fractions of $R$ (this is true for any right Ore domain; it follows e.g. from Prop. 3, p. 95 of [7]). The $R$-module $K$, as image of $I$, is again injective.

We can write $I$ in the form $I=\underline{\lim } a^{-1} R$; then

$$
\text { End }\left(K_{R}\right)=\operatorname{Hom}\left(\varliminf^{-1} R / R, K\right)=\varliminf \operatorname{Hom}\left(a^{-1} R / R, K\right)=\varliminf R / R a \text {, }
$$
hence

$$
\text { End }\left(K_{R}\right)=\varliminf \underline{\lim } R / R a=\hat{R} .
$$

Clearly $K$ is an injective cogenerator for $T_{R}^{\dagger}$; hence $\hat{R}$, defined by (4), is a projective generator for ${ }_{R} T^{\uparrow}$. In fact $\hat{R}$ is just the ring $E$ defined in (3), hence ${ }_{R} T^{\uparrow}$ may be regarded as the category of all pseudo-compact left $\hat{R}$-modules.

Since $K$ is a direct sum of indecomposable injectives (cf. e.g. [1], 6.50), $\hat{R}$ is a direct product of indecomposable projectives. These correspond to the different atoms of $R$ and the indecomposable projective corresponding to a given atom $p$ of $R$ has the form

$$
R_{p}=\varliminf R / R a,
$$

where $a$ runs over all elements of $R$ containing $p$ as essential right factor. In the commutative case (5) of course simplifies to

$$
R_{p}=\underline{\lim } R / R p^{n} .
$$

Returning to the case of a general fir $R$, we still have an injective cogenerator $K$ for $T_{R}^{\dagger}$ which is a direct sum of indecomposable injectives. Its dual $D K$ is a direct product of indecomposable projectives and is a projective generator for ${ }_{R} T^{\downarrow}$. However, there is now no natural way of constructing $D K$ (analogously to $\hat{R}$ ); firstly because not every simple object of $T_{R}$ is defined by an atom in $R$ (we generally need $R_{n}$ for all $n$ ), and secondly, because even in $R$ itself the family of principal right ideals generated by atoms is not closed under finite intersections. Even the formula (5) for an indecomposable projective may fail in this case: e.g., if $R=$ $k\langle x, y, z\rangle$ is the free $k$-algebra on $x, y, z$ ( $k$ a field), then $y x, z x$ are two elements with $x$ as essential right factor, but $R y x \cap R z x=0$. This means that the injective hull of $R / x R$ cannot be expressed as a direct limit of cyclic modules, and correspondingly the projective cover of $R / R x$ is not an inverse limit of the form (5). 


\section{References}

[1] I. Bucur and A. Deleanu, Categories and functors (London, New York, Sydney 1968).

[2] P. M. Cohn, 'Free ideal rings', J. Alg. 1 (1964) 47-69.

[3] P. M. Cohn, 'Torsion modules over free ideal rings', Proc. London Math. Soc. (3) 17 (1967) $577-599$.

[4] P. M. Cohn, 'Bound modules over hereditary rings', to appear.

[5] P. Gabriel, 'Des categories abeliennes', Bull. Soc. Math. France 90 (1962) 323-448.

[6] P. Gabriel and U. Oberst, 'Spektralkategorien und reguläre Ringe im von Neumannschen Sinn', Math. Zeits. 92 (1966) 389-395.

[7] J. Lambek, Lectures on rings and modules (Waltham Mass., Toronto and London 1966).

[8] J. E. Roos, 'Locally distributive categories and strongly regular rings', Reports of the Midwest Category Seminar (Springer Lecture Notes in Mathematics, No. 47).

\section{Bedford College}

Regents Park

London N.W. 1 